\title{
Heterogeneous impact of a lockdown on inter-municipality mobility
}

\author{
Hygor P. M. Melo $\odot,{ }^{1,}{ }^{*}$ João Henriques,,${ }^{2,3}$ Raquel Carvalho, ${ }^{2}$ Trivik Verma $\odot,{ }^{4}$ João P. da Cruz $\odot,{ }^{1,5,6,7}$ \\ and N. A. M. Araújo ${ }^{1,6, \dagger}$ \\ ${ }^{1}$ Centro de Física Teórica e Computacional, Faculdade de Ciências, Universidade de Lisboa, 1749-016 Lisboa, Portugal \\ ${ }^{2}$ NOS, SGPS, S.A., 1600-404 Lisboa, Portugal \\ ${ }^{3}$ Altran Portugal, 1990-096 Lisboa, Portugal \\ ${ }^{4}$ Faculty of Technology, Policy and Management, Delft University of Technology, 2628BX Delft, The Netherlands \\ ${ }^{5}$ Closer Consultoria, 1070-101 Lisboa, Portugal \\ ${ }^{6}$ Departamento de Física, Faculdade de Ciências, Universidade de Lisboa, 1749-016 Lisboa, Portugal \\ ${ }^{7}$ School for Data Science and Computational Thinking, Stellenbosch Un. 44 Banghoek Road, Stellenbosch, 7602, South Africa
}

(Received 28 June 2020; accepted 20 December 2020; published 13 January 2021)

\begin{abstract}
Without a vaccine, the fight against the spreading of the coronavirus has focused on maintaining physical distance. To study the impact of such measures on inter-municipality traffic, we analyze a mobile dataset with the daily flow of people in Portugal in March and April 2020. We find that the reduction in inter-municipality traffic depends strongly on its initial outflow. In municipalities where the mobility is low, the outflow reduced by 10-20\% and this decrease was independent of the population size. Whereas, for municipalities of high mobility, the reduction was a monotonic increasing function of the population size and it even exceeded $60 \%$ for the largest municipalities. As a consequence of such heterogeneities, there were significant structural changes on the most probable paths for the spreading of the virus, which must be considered when modeling the impact of control measures.
\end{abstract}

DOI: 10.1103/PhysRevResearch.3.013032

\section{INTRODUCTION}

In response to the coronavirus pandemic, many governments imposed lockdowns, closing several public places, such as, schools, restaurants, sport centers, and shopping malls [1]. Companies and public services have opted for giving employees the choice to work from home [2]. These nonpharmacological interventions are believed to be responsible for a significant slowdown of the spreading in different countries [3-10]. However, one still lacks an understanding on how the mobility patterns have changed and how such changes affect the most probable pathways for the spread of the virus.

With digitalization and advancements in global data collection, the public expectations for knowledge-based strategies to control the spreading of the coronavirus have been high. Daily, the public is swamped with statistics about new infections, deaths, and recoveries around the world [11,12]. Models and metrics are being proposed and further developed to turn data into actionable insights [13]. But statistics of demographic variations of the impact of a virus are not enough. An extensive body of research on recent public health threats, such as the 2013 MERS-CoV, 2014 Ebola, and 2016 Zika

\footnotetext{
*hpmelo@fc.ul.pt

${ }^{\dagger}$ nmaraujo@fc.ul.pt

Published by the American Physical Society under the terms of the Creative Commons Attribution 4.0 International license. Further distribution of this work must maintain attribution to the author(s) and the published article's title, journal citation, and DOI.
}

viruses, has shown that to estimate the spread and implement efficient actions for controlling it, one needs to rigorously investigate the mobility patterns [14-17]. Depending on the level of granularity, mobility data might be obtained from civil aviation statistics $[18,19]$, national censuses [19,20], public transit ridership [21-23], or mobile phone tracking [20,24]. By May 2020, there were more than 60k travel restrictions issued around the world to control inter- and intra-country traveling [25]. The mobility patterns today are very different from the ones in February 2020, and these publicly available datasets are no longer representative.

Epidemic spreading is a network-driven process [24,26-28]. To understand the complex spatiotemporal spreading dynamics, one needs to replace the traditional view based on geographical distance by a probabilistically motivated effective distance computed from the flux of people [29]. With such a change of paradigm, one can identify the set of most probable paths for the spreading of the virus and the relative arrival times of epidemics, which are both independent of the epidemic parameters. Thus, the role of a municipality on the global dynamics depends on its daily flow and effective distance to other municipalities.

Here, we study this impact by analyzing mobile phone data for the inter-municipality daily flow of people in Portugal in March and April 2020. The first confirmed case of COVID-19 in Portugal was reported on March 2, 2020. Ten days later, the total number of cases added up to 78 and the government announced a first set of control measures, which included school closures from March 14 onward. With a total of 642 identified cases, on March 19, the government decided to declare the state of emergency to impose more drastic measures 

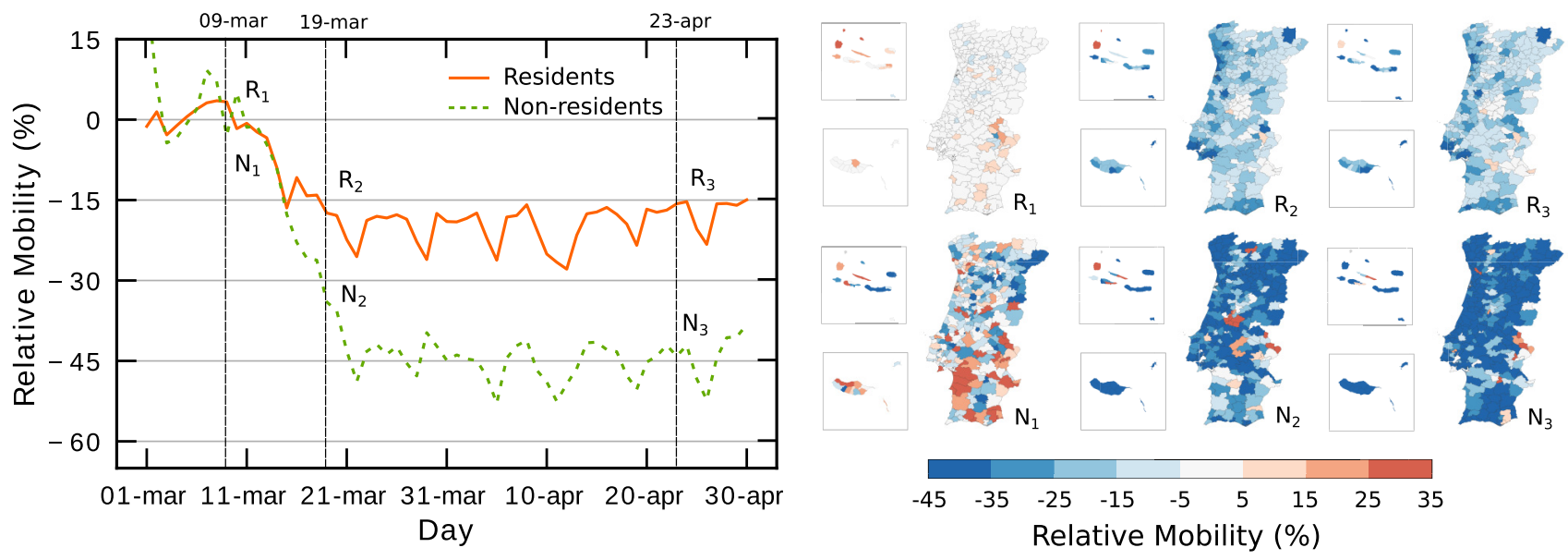

FIG. 1. Effect of the lockdown in the mobility pattern of residents and nonresidents in Portugal. The relative mobility is calculated as the total number of people that moved between two different municipalities on that day divided by the average between March 2 and 6 (see Sec. IV for further details). The first control measures were announced in March 12, and the state of emergency was declared on March 18. We see that during the lockdown the mobility of residents and nonresidents decreased to about $20 \%$ and $45 \%$ of the baseline. The maps show the spatial distribution of the relative mobility for three specific days (March 9, March 19, and April 23), for residents (R1, R2, and R3) and for nonresidents (N1, N2, and N3).

of mobility restriction and social distance. We show that the impact of the different measures was neither instantaneous nor homogeneous throughout the country. It extended over several days and the relative impact was strongly dependent on the population size of the municipality. We also analyze how the changes in mobility impacted on the distances. We show that the effective distances changed differently for each municipality. The structure of the set of most probable paths and relative arrival times also changed, with a significant impact on the spreading dynamics.

\section{RESULTS}

We analyze a national-scale mobile dataset provided by NOS, a Portuguese telecommunication company. This dataset contains the daily number of people that moved within and between municipalities in Portugal in March and April 2020, obtained from the mobile recurrent usage of residents and nonresidents (see Sec. IV for further details). The nonresidents are defined as users with a SIM card from a foreign mobile network. A user is registered in the dataset if they stay for, at least, 60 minutes within the boundaries of a municipality (see Sec. IV for further details).

To quantify the inter-municipality mobility, we measure the daily number of users moving within and between municipalities and define relative mobility as this number rescaled by the average mobility from March 2 to 6 (baseline), which is a period before any control measure. In Fig. 1, we show the time dependence of the relative mobility averaged over all municipalities for residents and nonresident users. Even before the declaration of the state of emergency (March 18), with the first set of control measures (March 12), the mobility started to decrease. However, this change in mobility was not instantaneous, it occurred over several days and only reached a steady level after March 21, with a decrease of about $20 \%$ for residents and $45 \%$ for nonresidents. The periodic structure observed in the steady regime corresponds to the expected weekday (Monday to Friday) and weekend (Saturday to Sunday) differences, with a clear decrease in mobility during the weekends. In the same figure, we show also the spatial distribution of the relative mobility for three different days (March 9, March 19, and April 23). One sees that the impact of the control measures was heterogeneous throughout the country. To characterize these heterogeneities, in what follows, we analyze the inter-municipality mobility. Since only $3.3 \%$ of the users are nonresidents, for simplicity, we consider only the resident one.

The origin-destination matrix (OD) is defined such that each element $P_{i j}(d)$ is the fraction of users that, in day $d$, stayed for more than 60 minutes in both municipalities $i$ and $j$ (see Sec. IV). From the OD matrix, we obtain the mobility network, which is a weighted directed graph, where the nodes are the municipalities and the weight of the link $i j$ is $P_{i j}$. Note that, in general, $P_{i j} \neq P_{j i}$, as the total number of users in each municipality is different (see Sec. IV for further details). The mobility network sets the stage for the propagation to other municipalities. As shown in Ref. [29], further insight into the propagation dynamics is obtained from the mobility network if we compute the effective distance $D_{\text {eff }}$ between nodes, defined as follows. For each pair of nodes $i$ and $j$, we defined the length of the link as $\ell_{i j}=1-\log \left(P_{i j}\right)$. Thus, the most probable path between two nodes $i j$ is the shortest path between the two, defined as the one that minimizes the sum of all $\ell$ along the path. The effective distance $D_{\text {eff }}^{i j}$ is then the value of such sum [29]. For every municipality $i$, we can obtain the shortest-path tree, consisting of the set of the most probable paths to all the other municipalities, where the central node is the origin $i$. In Fig. 2, we show three examples of the shortest-path trees, for Fig. 2(a) Lisbon, Fig. 2(b) Braga, and Fig. 2(c) Campo Maior, using an OD matrix obtained from the average mobility from March 2 to 6. All nodes are placed at a distance from the central node proportional to their effective distance to it. For the sake of comparison, the color of a node is given by the geographic 
(a)
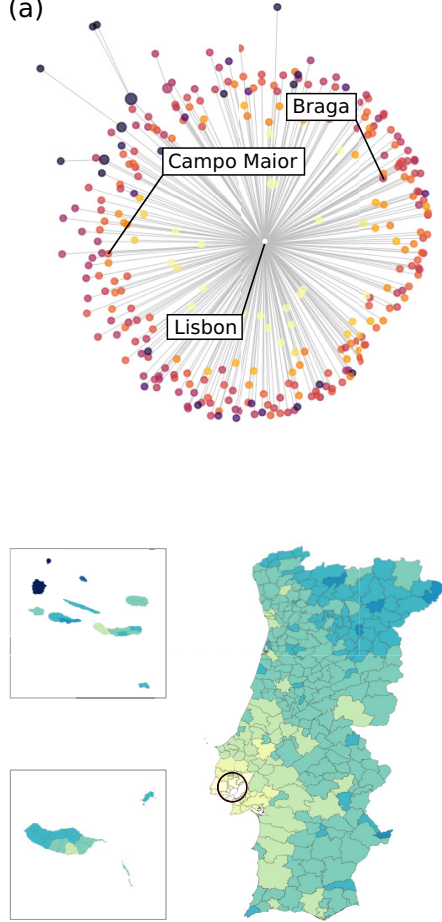

(b)
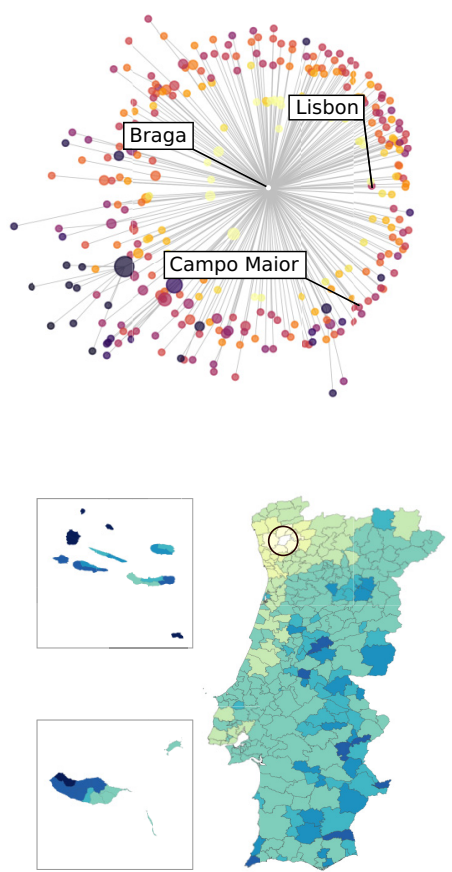

(c)

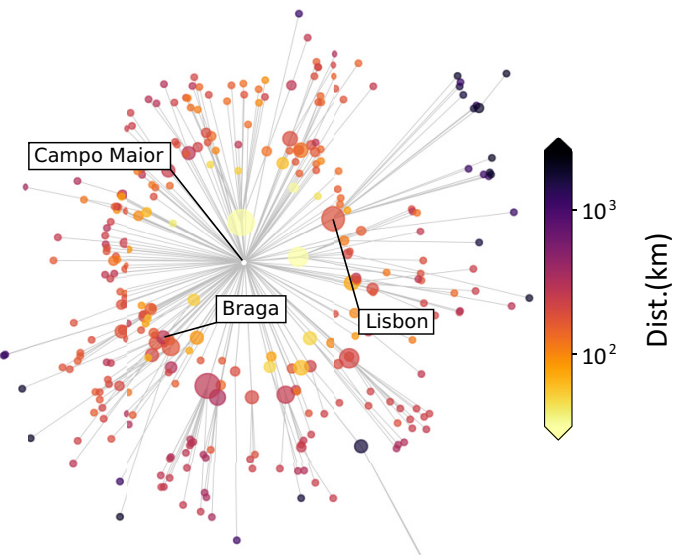

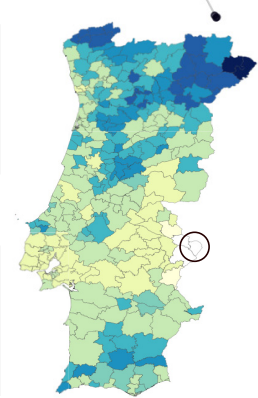

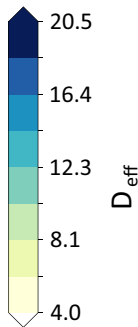

FIG. 2. Shortest-path trees and effective distances before the lockdown. Shortest-path trees from (a) Lisbon, (b) Braga, and (c) Campo Maior (central nodes). Each node is a different municipality and the distance to the central node is proportional the effective distance $D_{\text {eff }}$, as defined in Sec. IV. The effective distances are calculated from the origin-destination matrix of the average mobility between March 2 and 6. The nodes are colored according to the geographical distance to the central node. In the bottom row are the country maps, where the color of each municipality is given by the effective distance from the corresponding central municipality marked by a black circle.

distance from the central node. The size of the node is given by the value of the betweenness centrality, which is defined as the number of shortest paths that go from the central node to any other node on the tree through that node. In the figure, below each tree, we also show a color map displaying the spatial distribution of effective distances from each center. It is clear that some municipalities can be geographically far from the central municipality, but have a small value of effective distance, indicating that the relation between effective distances and geographic distances are nontrivial.

Since the change in mobility was heterogeneous throughout the country, as shown in Fig. 1, it is expected that both the effective distances and structure of the shortest-path trees changed. To illustrate such changes, we show in Fig. 3(a) the time evolution of the shortest-path tree for Lisbon. We discover a change from a star-like structure to a more branched structure. Figure 3(b) shows the time evolution of the average effective distance $\left\langle D_{\text {eff }}\right\rangle$ to all other municipalities for Lisbon, Braga, and Campo Maior. With the lockdown, $\left\langle D_{\text {eff }}\right\rangle$ increased, consistent with a much slower propagation dynamics. The maximum on the Easter holidays (gray region) is reflective of a set of additional measures imposed by the Portuguese government for those days to avoid the traditional family gatherings, which included severe restrictions to the inter-municipality mobility. For each day $d$, we calculated the Spearman's correlation between the effective distance $D_{\text {eff }}(d)$ and the initial effective distance $\overline{D_{\text {eff }}}$, obtained from the average mobility network from March 2 to 6 (before the lockdown). The Spearman's correlation $r$ for the entire set of municipalities is defined as

$$
r=\frac{\operatorname{cov}\left(\mathrm{rk}_{\mathrm{d}}, \overline{\mathrm{rk}}\right)}{\sigma\left(\mathrm{rk}_{\mathrm{d}}\right) \sigma(\overline{\mathrm{rk}})},
$$

where $\mathrm{rk}_{\mathrm{d}}$ is the rank of $D_{\text {eff }}(d), \overline{\mathrm{rk}}$ is the rank of $\overline{D_{\text {eff }}}$, $\operatorname{cov}\left(\mathrm{rk}_{\mathrm{d}}, \overline{\mathrm{rk}}\right)$ is the covariance of the rank, and $\sigma(\mathrm{rk})$ is the standard deviation. An $r=1.0$ means that the rank of effective distances has not changed, while $r=0$ corresponds to a new rank that is uncorrelated from the original one. The daily evolution of $r$ is shown in Fig. 3(c). For the three municipalities, $r$ decreased with the lockdown. This result suggests that the hierarchical organization of municipalities in a spreading process was affected significantly by the lockdown.

Figure 3(d) depicts the evolution of the degree $k$ of the central node (Lisbon, Braga, and Campo Maior), corresponding to the number of municipalities that are directly connected to the central node in the shortest-path tree. Before the lockdown, Lisbon was connected by a single edge to $90 \%$ of the municipalities. With the lockdown, Lisbon, on average, became only connected directly to $55 \%$ of the municipalities. This is in line with a change from a star-like to a branched structure, as observed in Fig. 3(a). We see a similar behavior for Braga, starting with the fraction of municipalities connected directly, decreasing from 58\% to 33\%, and for Campo Maior, where the drop is from $23 \%$ to $15 \%$.

In Fig. 4, we compare the outflow before and during the lockdown. The outflow is defined as the total number of 
(a)

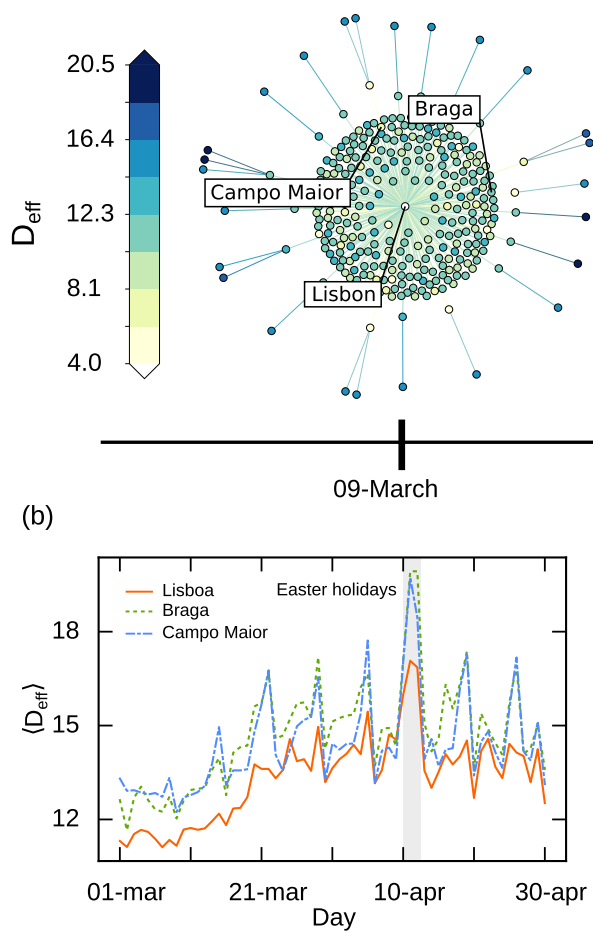

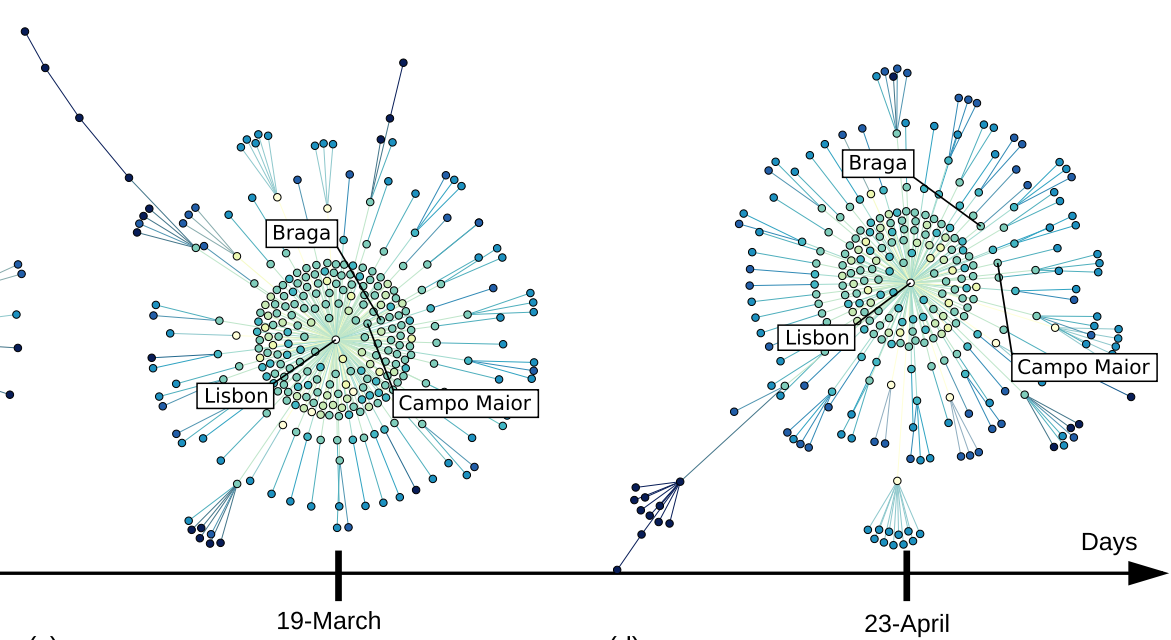

(c)

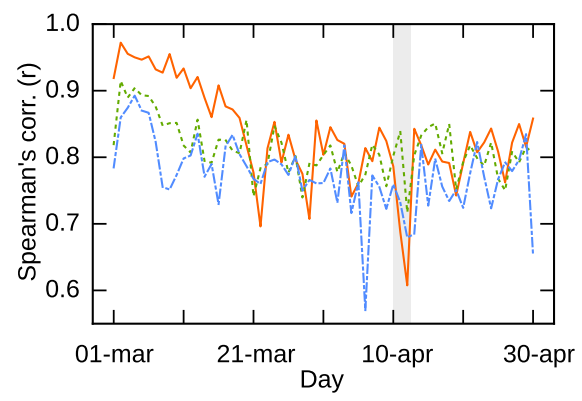

(d)

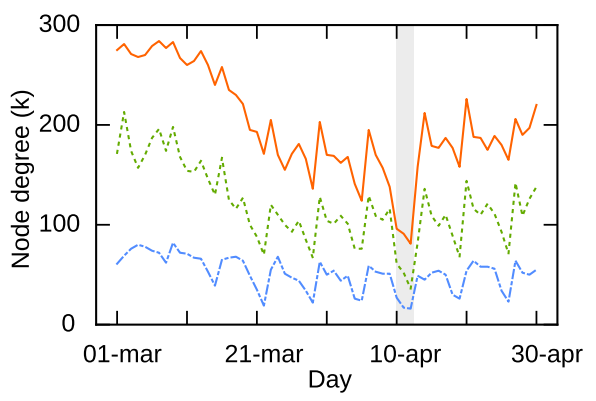

FIG. 3. The effect of the lockdown on the mobility network. (a) Shortest-path tree from Lisbon for three different days. The position of the nodes is given by the Fain Hu layout [30], designed to distribute symmetrically the nodes and minimize edge crossing. The color of each node is given by the effective distance from Lisbon. (b) Time dependence of the average effective distance in the shortest-path trees shown in Fig. 2. Before the lockdown, for these three municipalities, Lisbon had the shortest average distance to the rest of the country, followed by Braga and Campo Maior. With control measurements, the three average effective distances increased, reaching a maximum in the Easter holidays, when the Portuguese government imposed more severe mobility restrictions to inter-municipality travels. (c) Spearman's correlation coefficient $r$ between the effective distance from the average origin-destination matrix from March 2 to 6, and all the days of March and April. With the lockdown, the rank of the effective distances have changed as shown. (d) Time evolution of the node degree $k$ (number of edges) for Lisbon, Braga, and Campo Maior, for the shortest-path trees shown in Fig. 2. Initially, Lisbon was connected directly to more than $90 \%$ of the municipalities. With the lockdown, the $k$ decreases reaching a minimum at the Easter holidays, with only less than $50 \%$ of the municipalities connected with Lisbon by only one edge.

resident users of a municipality that were also identified in a different municipality during the day (see Sec. IV). The values before the lockdown are averages from March 2 to 6 and during the lockdown from April 20 to 24. We find that, although the control measures were the same for the entire country, their impact on the outflow strongly depends on the initial value of the outflow. For municipalities with low values of outflow, we observe a linear relation between the outflows before and after, which suggests that the relative impact is the same. However, for the municipalities with higher values of the outflow, we observe a sublinear relation between the two. As we show in the Supplemental Material [31], for the same period in 2019, the relation between the two outflows is linear, discarding any seasonal effects. The larger the initial value of the outflow the stronger is the impact of the control measures. Since the outflow of a municipality correlates strongly with the population size [33], in the inset of Fig. 4, we plot the relative decrease in outflow as a function of the population size. For municipalities with less than 18000 users, the outflow decreased by 10-20\%, without a clear dependence on the population size. For larger municipalities, the relative decrease in the outflow scales logarithmically with the population size (black dashed line) and it exceeds $60 \%$ for the largest municipality (Lisbon). Another variable that could explain the variation on the relative outflow is the spatial extension of the municipalities. However, as we show in the Supplemental Material [31], the area is not enough to explain the impact of the lockdown on mobility.

\section{CONCLUSION}

Human mobility sets the stage for global spreading phenomena [19,27,29,34,35]. Across the world, the most relevant nonpharmacological interventions to hinder the spreading of the coronavirus have been related to closing borders and restricting the intra- and inter-country mobility, which has affected the mobility patterns [9]. The change in the mobility patterns could lead to different responses on the propagation of a virus, as shown in Spain [36,37], however, the mobility reduction was shown to be, in general, correlated with an effective reduction of the contagion [5,38]. Here, we studied the impact of such mobility restrictions in the inter- 


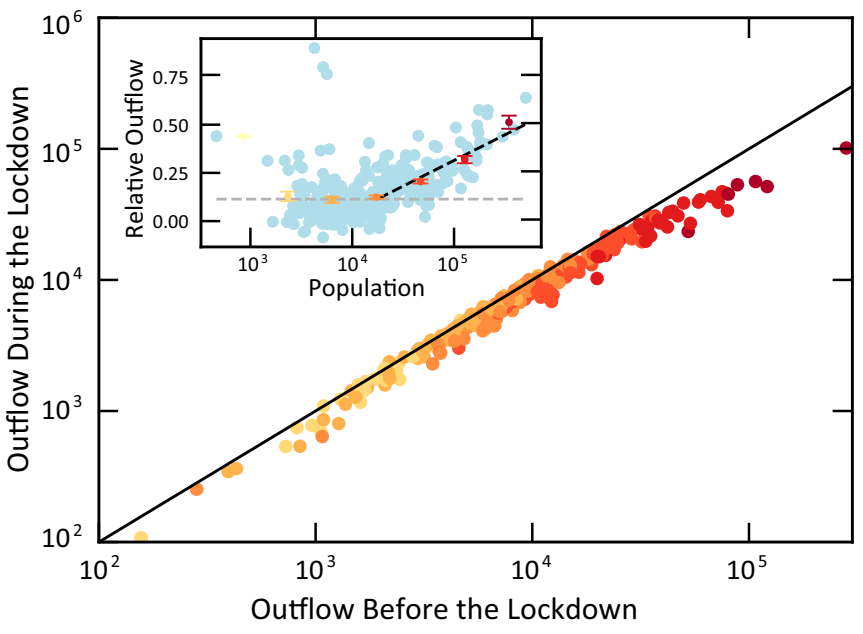

FIG. 4. The impact of the lockdown depends on the mobility pattern of the municipality. Outflow of each municipality before and during the lockdown. To reduce the statistical noise, the outflow corresponds to averages over five days: before any control measurements, we considered March 2 to 6, and for during the lockdown April 22 to 26. We show in the Supplemental Material [31] that a similar result is obtained when averaging over 10 days. Each colored circle is a municipality and the black solid line is a guide for the eyes and corresponds to a linear relation between the two. The color of each data point represents the population size of the municipality, following the log-spaced bins shown in the inset. We can see that the effect of the lockdown is not homogeneous across the country, municipalities with large values of mobility at the beginning of March showed a large reduction in April. In the inset is the relative change on outflow of each municipality (cyan circles) as a function of its population size. The relative change is calculated by the outflow difference between March and April divided by the one in March. The colors are given by the average relative change on outflow in log-spaced bins (based on the population size). The gray dashed line shows the average value of the relative outflow for the smaller municipalities ( $<1800$ people), and the dashed black line is the best fit for a logarithmic dependence of the relative outflow (RO) on the population size $(P)$ for large municipalities $(>18000$ people) $\mathrm{RO}=0.27 \log _{10}(P)-1.02$, while for smaller municipalities the best fit is $\mathrm{RO}=-0.06 \log _{10}(P)+0.33$. The population for each municipality was obtained from Ref. [32].

municipality flow of people in Portugal, in March and April of 2020. From a large dataset of mobile phone users, we obtained the origin-destination matrix and the shortest-path trees from all municipalities. While local mobility and social activity are important to understand the growth of the epidemic, the shortest-path trees between municipalities are what define the time sequence of municipalities affected and capture the most probable paths for the spread of the virus, starting at a given municipality $[24,29,39]$. We find that the relative decrease in mobility correlates with the population size of a municipality. For small municipalities, with less than 18000 mobile users, the outflow is decreased by about 10-20\%, regardless of the size of the municipality. However, for larger municipalities, the relative decrease in the outflow depended strongly on the population size and it even exceeds $60 \%$ for the largest municipalities. The change in the mobility network demonstrates that not only does the lockdown promote a delay in the virus propagation, but also deeply affects the structure of the shortest-path trees and, therefore, changed the temporal sequence of affected municipalities.

To circumvent the absence of accurate data for mobility, models were developed to artificially generate them, as, for example, the gravity and radiation models $[33,40]$, or to account for mobility restrictions in the models $[28,41,42]$. From our findings, it is now possible to extend these models to account for the dependence on the size and mobility of a municipality. This heterogeneous response is an important ingredient to consider in epidemic models, such as the ones developed in Refs. [28,41-49], so that governments can respond to the spread more accurately.

\section{MATERIALS AND METHODS}

\section{A. Mobility matrix from mobile phones records}

The mobile data was obtained from NOS cellular network and its volume goes up to about 8 Terabytes of events, collected from the network interfaces in March and April 2020. Each record in the dataset refers to a unique subscriber id, General Data Protection Regulation (GDPR) compliant regarding Personal Identifiable Information (PII) [50], and it includes the date/time-stamp of each respective event and the geographic coordinates of the respective network cell coverage centroid where each event occurred. Such a dataset can thus provide an abstraction of subscriber physical displacements over time.

The process to compute the mobility matrices is composed of several steps: from data event aggregation and location estimation to trip count aggregation. Within the data event aggregation part, the events are aggregated in a 10 minute sliding window with detection counts for each subscriber id performed by cell. The location for each subscriber id is estimated based on the most frequent cell algorithm. Once the location trips are extracted, the process to derive origindestination flows between geographic areas is the following. (i) The geographic area under analysis is divided into municipalities. (ii) The origin and destination municipalities, together with starting time, are extracted for each subscriber id. (iii) Trips with the same origin, destination, and time frame are grouped together. The result is a matrix whose element represents the number of trips from origin $i$ to destination $j$, starting within the time frame. We used the 308 municipal areas in Portugal for the regions and the time frame of 6 am and $8 \mathrm{pm}$. The level of municipality was considered because, to tackle health inequalities, policy interventions are often mandated from national governments to each municipality $[51,52]$.

It was observed that the mobile data are a good proxy for the mobility matrix: although there is a tendency to overestimate the number of commuters, the calculation of the shortest-path tree is preserved when compared with census data [24].

From the mobility matrix, the extrapolation process is carried out to represent the whole population in study. The extrapolation algorithm takes into consideration local as well as international market shares (in 2020, the company holds $26 \%$ of the market share in Portugal), besides wholesale 
agreements between parties. After extrapolation, the transformation process takes place to extract features from residents and nonresidents, segmenting the data between flows from region $i$ to $j$, and the ones that do not move within that time window, which is the same as saying that $i$ is equal to $j$. At the end, compliance with the data privacy rules (GDPR) are applied and trips with counts under six are omitted.

\section{B. Shortest-path trees}

For the network analysis, we only considered the movement of residents, i.e., in the mobility matrix $\mathbf{F}(\mathbf{d}), F_{i j}(d)$ is the number of resident users who were identified at both municipality $i$ and $j$ within the day $d$. To measure the combined number of individuals from $i$ to $j$, we considered the undirected mobility network using the standard symmetrization operation $\mathbf{F}=\mathbf{F}+\mathbf{F}^{T}$, where $\mathbf{F}^{T}$ is the transposed matrix of F [29].

[1] T. Hale, A. Petherick, T. Phillips, and S. Webster, Variation in government responses to covid-19, Blavatnik school of government working paper BSG-WP-2020/032 (2020).

[2] E. Brynjolfsson, J. J. Horton, A. Ozimek, D. Rock, G. Sharma, and H. TuYe, Covid-19 and remote work: An early look at US data, Working Paper 27344 (National Bureau of Economic Research, Cambridge, MA, 2020), https://www.nber.org/system/ files/working_papers/w27344/w27344.pdf.

[3] B. F. Maier and D. Brockmann, Effective containment explains subexponential growth in recent confirmed covid-19 cases in china, Science 368, 742 (2020).

[4] J. Zhang, M. Litvinova, Y. Liang, Y. Wang, W. Wang, S. Zhao, Q. Wu, S. Merler, C. Viboud, A. Vespignani, M. Ajelli, and $\mathrm{H}$. Yu, Changes in contact patterns shape the dynamics of the covid-19 outbreak in China, Science 368, 1481 (2020).

[5] M. U. G. Kraemer, C. Yang, B. Gutierrez, C. Wu, B. Klein, D. M. Pigott, L. Du Plessis, N. R. Faria, R. Li, W. P. Hanage, J. S. Brownstein, M. Layan, A. Vespignani, H. Tian, C. Dye, O. G. Pybus, and S. V. Scarpino, The effect of human mobility and control measures on the covid-19 epidemic in china, Science 368, 493 (2020).

[6] C. O. Buckee, S. Balsari, J. Chan, M. Crosas, F. Dominici, U. Gasser, Y. H. Grad, B. Grenfell, M. E. Halloran, M. U. G. Kraemer, M. Lipsitch, C. Jessica, E. Metcalf, L. Ancel Meyers, T. A. Perkins, M. Santillana, S. V. Scarpino, C. Viboud, A. Wesolowski, and A. Schroeder, Aggregated mobility data could help fight COVID-19, Science 368, 145 (2020).

[7] A. Remuzzi and G. Remuzzi, Covid-19 and Italy: what next? The Lancet 395, 1225 (2020).

[8] G. Giordano, F. Blanchini, R. Bruno, P. Colaneri, A. Di Filippo, A. Di Matteo, and M. Colaneri, Modelling the covid-19 epidemic and implementation of population-wide interventions in italy, Nature Medicine 26, 855 (2020).

[9] H. Tian, Y. Liu, Y. Li, C. Wu, B. Chen, M. U. G. Kraemer, B. Li, J. Cai, B. Xu, Q. Yang, B. Wang, P. Yang, Y. Cui, Y. Song, P. Zheng, Q. Wang, O. N. Bjornstad, R. Yang, B. T. Grenfell, O. G. Pybus, and C. Dye, An investigation of transmission control measures during the first 50 days of the covid-19 epidemic in china, Science 368, 638 (2020).
Each element of the origin-destination matrix $\mathbf{P}$ is calculated as $P_{i j}=F_{i j} / \sum_{n} F_{i n}$, where the sum is over all municipalities, and quantifies the mobility network, where the nodes are the municipalities and the edges between $i$ and $j$ have a weight $P_{i j}$. If we define the distance $\ell_{i j}=1-\log \left(P_{i j}\right)$ for each edge, the most probable paths are the ones that minimize the total sum of $\ell$ [29]. For each node, one can define a shortest-path tree (see Fig. 2) and the effective distance $D_{\text {eff }}$ from a node $i$ to $j$ as the length of the shortest path between these two nodes.

\section{ACKNOWLEDGMENTS}

We acknowledge financial support from the Portuguese Foundation for Science and Technology (FCT) under Contracts No. PTDC/FIS-MAC/28146/2017(LISBOA01-0145-FEDER-028146), UIDB/00618/2020, and UIDP/00618/2020, and from NOS SGPS S.A. for providing the mobile data.
[10] H. Salje, C. Tran Kiem, N. Lefrancq, N. Courtejoie, P. Bosetti, J. Paireau, A. Andronico, N. Hozé, J. Richet, C. Dubost, Y. Le Strat, J. Lessler, D. Levy Bruhl, A. Fontanet, L. Opatowski, P.Y. Boelle, and S. Cauchemez, Estimating the burden of sarscov-2 in France, Science 369, 208 (2020).

[11] E. Dong, H. Du, and L. Gardner, An interactive web-based dashboard to track covid-19 in real time, Lancet Infect. Dis. 20, 533 (2020).

[12] Financial Times, Coronavirus Tracked: The Latest Figures as Countries Fight to Contain the Pandemic, from: https://www.ft. com/content/a26fbf7e-48f8-11ea-aeb3-955839e06441. date of access 22 Jun 2020.

[13] A. P. y Piontti, N. Perra, L. Rossi, N. Samay, and A. Vespignani, Charting the Next Pandemic: Modeling Infectious Disease Spreading in the Data Science Age (Springer, New York, 2018).

[14] M. E. Halloran, A. Vespignani, N. Bharti, L. R. Feldstein, K. A. Alexander, M. Ferrari, J. Shaman, J. M. Drake, T. Porco, J. N. S. Eisenberg, S. Y. Del Valle, E. Lofgren, S. V. Scarpino, M. C. Eisenberg, D. Gao, J. M. Hyman, S. Eubank, and I. M. Longini, Jr., Ebola: mobility data, Science 346, 433 (2014).

[15] A. Wesolowski, C. O. Buckee, L. Bengtsson, E. Wetter, X. $\mathrm{Lu}$, and A. J. Tatem, Commentary: containing the Ebola outbreak - the potential and challenge of mobile network data, PLOS Curr. Outbreaks (2014), doi: 10.1371/currents.outbreaks. 0177e7fcf52217b8b634376e2f3efc5e

[16] C. Poletto, C. Pelat, D. Lévy-Bruhl, Y. Yazdanpanah, P. Y. Boëlle, and V. Colizza, Assessment of the Middle East respiratory syndrome coronavirus (MERS-CoV epidemic in the Middle East and risk of international spread using a novel maximum likelihood analysis approach, Eurosurveillance 19, 20824 (2014).

[17] I. I. Bogoch, O. J. Brady, M. U. G. Kraemer, M. German, M. I. Creatore, M. A. Kulkarni, J. S. Brownstein, S. R. Mekaru, S. I. Hay, E. Groot, A. Watts, and K. Khan, Anticipating the international spread of zika virus from brazil, The Lancet $\mathbf{3 8 7}$, 335 (2016).

[18] O. Woolley-Meza, C. Thiemann, D. Grady, J. J. Lee, H. Seebens, B. Blasius, and D. Brockmann, Complexity in human transportation networks: a comparative analysis of worldwide 
air transportation and global cargo-ship movements, Eur. Phys. J. B 84, 589 (2011).

[19] H. Barbosa, M. Barthelemy, G. Ghoshal, C. R. James, M. Lenormand, T. Louail, R. Menezes, J. J. Ramasco, F. Simini, and M. Tomasini, Human mobility: Models and applications, Phys. Rep. 734, 1 (2018).

[20] M. C. Gonzalez, C. A. Hidalgo, and A. Barabasi, Understanding individual human mobility patterns, Nature 453, 779 (2008).

[21] C. Ponte, H. P. M. Melo, C. Caminha, J. S. Andrade, and V. Furtado, Traveling heterogeneity in public transportation, EPJ Data Science 7, 42 (2018).

[22] C. Caminha, V. Furtado, T. H. C. Pequeno, C. Ponte, H. P. M. Melo, E. A. Oliveira, and J. S. Andrade, Human mobility in large cities as a proxy for crime, PLoS ONE 12, e0171609 (2017).

[23] Trivik Verma, Mikhail Sirenko, Itto Kornecki, Scott Cunningham, and Nuno AM Araújo, Extracting spatiotemporal demand for public transit from mobility data, arXiv:2006.03351.

[24] M. Tizzoni, P. Bajardi, A. Decuyper, G. K. K. King, C. M. Schneider, V. Blondel, Z. Smoreda, M. C. González, and V. Colizza, On the use of human mobility proxies for modeling epidemics, PLoS Comput. Biol. 10, e1003716 (2014).

[25] The UN Migration Agency, DTM (COVID-19) Global Mobility Restriction Overview, 07 May 2020, https://dtm.iom.int/reports.

[26] R. Pastor-Satorras, C. Castellano, P. Van Mieghem, and A. Vespignani, Epidemic processes in complex networks, Rev. Mod. Phys. 87, 925 (2015).

[27] J. Gómez-Gardenes, D. Soriano-Panos, and A. Arenas, Critical regimes driven by recurrent mobility patterns of reactiondiffusion processes in networks, Nat. Phys. 14, 391 (2018).

[28] S. Meloni, N. Perra, A. Arenas, S. Gómez, Y. Moreno, and A. Vespignani, Modeling human mobility responses to the largescale spreading of infectious diseases, Sci. Rep. 1, 62 (2011).

[29] D. Brockmann and D. Helbing, The hidden geometry of complex, network-driven contagion phenomena, Science 342, 1337 (2013).

[30] Y. Hu, Efficient, high-quality force-directed graph drawing, Mathematica Journal 10, 1 (2006).

[31] See Supplemental Material at http://link.aps.org/supplemental/ 10.1103/PhysRevResearch.3.013032 for more information about the results using 10 days average.

[32] Dataset for the population of each municipality in Portugal in 2018, from https://www.pordata.pt/Tema/Municipios.

[33] F. Simini, M. C. González, A. Maritan, and A. Barabási, A universal model for mobility and migration patterns, Nature 484, 96 (2012).

[34] R. Guimera, S. Mossa, A. Turtschi, and L. A. N. Amaral, The worldwide air transportation network: Anomalous centrality, community structure, and cities' global roles, Proc. Natl. Acad. Sci. 102, 7794 (2005).

[35] M. Lee, H. Barbosa, H. Youn, P. Holme, and G. Ghoshal, Morphology of travel routes and the organization of cities, Nat. Commun. 8, 2229 (2017).

[36] M. Mazzoli, D. Mateo, A. Hernando, S. Meloni, and J. J. Ramasco, Effects of mobility and multi-seeding on the propagation of the covid-19 in Spain, medRxiv (2020).

[37] J. Aguilar, A. Bassolas, G. Ghoshal, S. Hazarie, A. Kirkley, M. Mazzoli, S. Meloni, S. Mimar, V. Nicosia, J. J. Ramasco, and A. Sadilek, Impact of urban structure on covid-19 spread, arXiv:2007.15367.

[38] P. Bonato, P. Cintia, F. Fabbri, D. Fadda, F. Giannotti, P. L. Lopalco, S. Mazzilli, M. Nanni, L. Pappalardo, D. Pedreschi, F. Penone, S. Rinzivillo, G. Rossetti, M. Savarese, and L. Tavoschi, Mobile phone data analytics against the covid-19 epidemics in italy: flow diversity and local job markets during the national lockdown, arXiv:2004.11278.

[39] A. P. Y. Piontti, M. F. C. Gomes, N. Samay, N. Perra, and A. Vespignani, The infection tree of global epidemics, Network Science 2, 132 (2014).

[40] J. de Dios Ortúzar and L. G. Willumsen, Modelling Transport (John Wiley \& Sons, New York, 2011).

[41] A. Arenas, W. Cota, J. Gmez-Gardenes, S. Gmez, C. Granell, J. T. Matamalas, D. Soriano-Panos, and B. Steinegger, Modeling the Spatiotemporal Epidemic Spreading of COVID-19 and the Impact of Mobility and Social Distancing Interventions, Phys. Rev. X 10, 041055 (2020).

[42] G. S Costa, W. Cota, and S. C. Ferreira, Outbreak diversity in epidemic waves propagating through distinct geographical scales, Phys. Rev. Research 2, 043306 (2020).

[43] L. Wang and X. Li, Spatial epidemiology of networked metapopulation: An overview, Chinese Science Bulletin 59, 3511 (2014).

[44] T. D. Hollingsworth, N. M. Ferguson, and R. M. Anderson, Will travel restrictions control the international spread of pandemic influenza? Nature medicine 12, 497 (2006).

[45] B. S. Cooper, R. J. Pitman, W. J. Edmunds, and N. J. Gay, Delaying the international spread of pandemic influenza, PLoS Medicine 3, e212 (2006).

[46] P. Bajardi, C. Poletto, J. J. Ramasco, M. Tizzoni, V. Colizza, and A. Vespignani, Human mobility networks, travel restrictions, and the global spread of 2009 h1n1 pandemic, PLoS ONE 6, e16591 (2011).

[47] N. M. Ferguson, D. A. T. Cummings, C. Fraser, J. C. Cajka, P. C. Cooley, and D. S. Burke, Strategies for mitigating an influenza pandemic, Nature 442, 448 (2006).

[48] V. Colizza, A. Barrat, M. Barthelemy, A.-J. Valleron, and A. Vespignani, Modeling the worldwide spread of pandemic influenza: baseline case and containment interventions, PLoS Medicine 4, e13 (2007).

[49] L. Wang, Y. Zhang, T. Huang, and X. Li, Estimating the value of containment strategies in delaying the arrival time of an influenza pandemic: A case study of travel restriction and patient isolation, Phys. Rev. E 86, 032901 (2012).

[50] EU General Data Protection Regulation (GDPR): Regulation (EU) 2016/679 of the European Parliament and of the Council of 27 April 2016 on the protection of natural persons with regard to the processing of personal data and on the free movement of such data, and repealing Directive 95/46/EC (General Data Protection Regulation), OJ 2016 L 119/1.

[51] D. Byrne, Complexity theory and planning theory: A necessary encounter, Planning Theory 2, 171 (2003).

[52] P. A. Collins and M. V. Hayes, The role of urban municipal governments in reducing health inequities: A meta-narrative mapping analysis, International Journal for Equity in Health $\mathbf{9}$, 13 (2010). 